\title{
Pharmacophore
}

ISSN-2229-5402

Journal home page: http://www.pharmacophorejournal.com

\section{AN OVERVIEW OF DIAGNOSIS AND MANAGEMENT OF OSTEOARTHRITIS IN PRIMARY HEALTH CARE CENTER: LITERATURE REVIEW}

\author{
Almuzini Saleh Ahmad S ${ }^{1}$, Hasan Mohammed Alshami², Shahad Bandar Almeqbel ${ }^{3}$, \\ Munirah Abdulaziz Bin Talib ${ }^{3 *}$, Mashael Abdulrahman Alshowaier ${ }^{3}$, Ali Khalid \\ Aljifry $^{4}$, Raef Adel Alturkistani ${ }^{5}$, Fatimah Fouad Albadrani ${ }^{6}$, Baraa Khaled \\ Alsamman $^{7}$, Muath Abdullah Alhashim ${ }^{8}$, Hassan Salem alsharyah' \\ 1. General Practitioner, Shubra PHC, Riyadh, KSA. \\ 2. Faculty of Medicine, Batterjy Medical College, Jeddah, KSA. \\ 3. Faculty of Medicine, Alfaisal University, Riyadh, KSA. \\ 4. Faculty of Medicine, Alfarabi Medical College, Riyadh, KSA. \\ 5. Faculty of Medicine, King Abdulaziz University, Jeddah, KSA. \\ 6. Faculty of Medicine, Imam Abdulrahman bin Faisal University, Dammam, KSA. \\ 7. Faculty of Medicine, Ibn Sina National College of Medicine, Jeddah, KSA. \\ 8. Internal Medicine Department, King Fahad hospital, Al Ahsa, KSA. \\ 9. Cardiac Surgery Department, National Guard Hospital, Riyadh, KSA
}

\section{ART ICLE INFO}

\section{Received:}

15 Nov 2020

Received in revised form:

21 Dec 2020

Accepted:

22 Dec 2020

Available online:

28 Dec 2020

Keywords: Osteoarthritis, Clinical

Features, Diagnosis, Management

\author{
A B S T R A C T
}

To Cite This Article: Almuzini Saleh Ahmad S, Hasan Mohammed Alshami, Shahad Bandar Almeqbel, Munirah Abdulaziz Bin Talib, Mashael Abdulrahman Alshowaier, Ali Khalid Aljifry and et al., (2020), "An Overview of Diagnosis and Management of Osteoarthritis in Primary Health Care Center: Literature Review", Pharmacophore, 11(6), 152-156.

Introduction

A case of osteoarthritis is to be considered whenever a family medicine doctor is presented with a joint pain case in a primary health care setting. [1-4] Generally, the patient's pain may include upper limb joints like shoulders and fingers, with lower limb joints like the hips, knees, and ankles. These presentations are usually in people in their forties or older, and the clinical presentation may vary in older cases. [5] Patients older than 50-years-old with this disease might commonly present with mild morning stiffness that progresses in severity as activity increases, along with arthralgia and movement limitations. For confirmation of this diagnosis, the use of radiographic diagnostic testing could prove of great help. Although the radiological outcomes moreover than not are not in line with the presentation of the patient's symptoms.

Osteoarthritis more commonly afflicts females, increasing with age progression. [6] Importantly, in the epidemiology of the osteoarthritic disease, the prevalence would be observed to be higher when screening is done through radiographic means. Osteoarthritis is commonly found in the joints of the hands, knees, and hips. While knee arthropathy occurs more in females,

Corresponding Author: Munirah Abdulaziz Bin Talib; Faculty of Medicine, Alfaisal University, Riyadh, KSA. Email: Muneerahaziz@gmail.com 
gender discrepancy does not often occur in other joints. [7] Furthermore, the female gender is a risk factor for osteoarthritis development, and there were reports on genetic predisposition with dietary factors.

Considering the prevalence above in a primary care setting, a common condition that might be presented is osteoarthritis. In this article, we will discuss how a family medicine doctor should approach a suspected osteoarthritis case, how to diagnose, and how to manage these patients.

\section{Methodology}

We searched Pubmed for ((Osteoarthritis) (AND (management) OR (diagnosis))). In regards to the inclusion criteria, the articles were selected based on the inclusion of one of the following topics; osteoarthritis, differential, diagnostic imaging, management, and pain control. Exclusion criteria were all other articles that did not have one of these topics as their primary endpoint.

\section{Review:}

A normal healthy joint consists of two bones, each with their layer of articular cartilage, which is a type of connective tissue that does not allow the friction of the two bones involved making it easier to glide against each other dynamically in weightbearing joints.

joints have different types, one of which is a synovial joint that consists of synovium and articular cartilage. The synovium consists of connective tissue, blood vessels, lymphatic vessels and two types of cells A and B. Type A cells clears debris, and type $\mathrm{B}$ is in charge of the production of synovial fluid that helps lubricate the two articular surfaces. as for the articular cartilage maintaining its health is the chondrocytes' job. Chondrocytes are embedded in an extracellular matrix with two major components, type II collagen and proteoglycans that both provide tensile strength and elasticity of the cartilage. Maintenance of the health of cartilage is by the process of breaking down old cartilage and producing new one instead.

\section{Pathophysiology}

Osteoarthritis occurs when there is the loss of articular cartilage combined with underlying defective repair mechanisms. Certain risk factors of osteoarthritis cause the scale to tip towards the catabolic activity in cartilage homeostasis regulated by chondrocytes. Inevitably leading to increased articular damage and decreased production of new cartilage. This increase in articular damage causes chondrocytes to compensate for damage by inciting a decrease in proteoglycans production and an increase in type II collagen, subsequently switching to type I collagen causing no interaction between it and proteoglycans. This latter shift in collagenous formation will lead to a decrease in elasticity of the cartilage causing further breakdown. Hence, this articular cartilage breakdown causes inflammation of the synovium. Eventually, bone friction occurs due to loss of cartilage that will cause bony eburnation and osteophytes formation which is a new bone formation with outward growth. [8]

\section{Risk Factors}

Multiple risk factors are modifiable, including being overweight or obese, working a job that necessitates squatting or bending, and playing sports that involve a lot of repetitive impacts. Non-modifiable risk factors include old age (more than 50-years-old), suffering a joint injury, or having a family history of osteoarthritis. Certain risk factors are further associated with debilitating osteoarthritis, these include female gender, presence of other comorbid conditions, and patients who had osteoarthritis for a long time. [9]

\section{Clinical Presentation}

Patients often present in their fifth decade with pain in the hands, hips, and knee joints, an alert physician should have a low threshold for suspecting osteoarthritis in such an inflammatory presentation. Common clinical manifestations of osteoarthritis include pain and swelling of joints with the presence of crepitations. This joint pain often would exacerbate during the evening period and alleviates by ample rest.

Along with that joint pain, patients who are 50 years and older, will usually have some movement limitations and morning stiffness that worsens throughout the day. Hence, differentiating it from rheumatoid arthritis. A commonly misdiagnosed illness in osteoarthritic patients is rheumatoid arthritis, the family physician should be able to differentiate both using clinical examination and laboratory findings in difficult cases (Table 1).

Table 1: Differentiating Osteoarthritic patients from Rheumatoid arthritic patients

\begin{tabular}{|c|c|c|}
\hline & Osteoarthritis & Rheumatoid Arthritis \\
\hline Joints affected & Distal interphalangeal, carpometacarpal joints, & $\begin{array}{c}\text { Proximal interphalangeal and } \\
\text { metacarpophalangeal joints }\end{array}$ \\
\hline Examination & Heberden's nodes & Soft warm and tender (inflamed) joints \\
\hline Joint stiffness & Hard stony bone-like joints & Worst after rest \\
\hline
\end{tabular}




\begin{tabular}{|c|c|c|}
\hline \multicolumn{2}{|c|}{ Pharmacophore, 11(6) 2020, Pages 152-156 } \\
\hline $\begin{array}{c}\text { Positive laboratory } \\
\text { investigation }\end{array}$ & $\begin{array}{c}\text { Negative Rheumatoid factor, anti-cyclic citrullinated } \\
\text { antibody, Normal ESR }\end{array}$ & $\begin{array}{c}\text { Positive Rheumatoid factor, anti-cyclic } \\
\text { citrullinated antibody, High ESR with or without } \\
\text { high C-reactive protein }\end{array}$ \\
\hline
\end{tabular}

Moreover, many differentials shall be in the mind of the physician and be ruled out in the clinical setting, including; traumatic injury, collagen vascular diseases, gout, and pseudogout arthropathies. Moreover, in patients with an active sexual history, a diagnosis of septic arthritis, especially in a limping patient, should be considered and ruled out. Further extradermatological manifestations include psoriasis, which presents as silvery scales on extensor surfaces. Psoriatic arthritis may as well present with painful joints associated with skin scaling.

\section{Diagnostic Imaging}

In patients presented with osteoarthritis risk factors and symptoms, the family physician should rely on clinical judgment before proceeding to image modalities. This is because most patients will have classical signs and symptoms at presentation, thus, the radiographic findings are often unnecessary for diagnosis. Nevertheless, in patients with ambiguous symptoms radiology may help and is still considered the gold standard for diagnosing osteoarthritis. Generally, the x-ray is done initially in these patients, with common findings including; narrowing of joint spaces, subchondral sclerosis, and osteophytes. The Kellgren Lawrence osteoarthritis classification criteria are commonly used to stage inflammatory damage based on the imaging findings of the affected joints (Table 2). [10]

Table 2: Kellgren Lawrence Osteoarthritis Classification Criteria

\begin{tabular}{|c|c|c|}
\hline Grade & Disease Status & Radiological Findings \\
\hline Grade 1 & Doubtful Diagnosis & Definite osteophyte formation \\
\hline Grade 2 & Mild disease & Moderate joint space reduction \\
\hline Grade 3 & Moderate disease & Great reduction in joint space, Subchondral sclerosis \\
\hline Grade 4 & Severe disease & \\
\hline
\end{tabular}

In addition to the diagnosis of difficult cases of osteoarthritis, radiographic imaging can be used to estimate inflammatory damage, and monitor treatment thereafter. Before referring a patient for replacement of the affected joint, radiography can be beneficial to check out the severity which is a determining factor to perform surgery or not. In most non-arthralgic patients, who are more than 50-years-old, magnetic resonance imaging would be useful in detecting abnormalities within the joint. [11]

\section{Management Approach}

A patient-centered approach is important in the management of this painful joint condition. In primary care practice, management should be done in a stepwise fashion beginning with conservative and medical therapy, while reserving complicated cases for surgical referral. For instance, exercising and losing weight are of great benefits in the knee and hip osteoarthritis. Medical treatment helps with reducing the patient's pain and improving his movement. Although opioids and non-steroidal anti-inflammatory drugs have shown to cause serious side effects.

\section{Conservative Therapy}

The choice for non-invasive, conservative management is regarded as a must in these patients. Nevertheless, the effect of these will be affected by which joint is affected, the severity of the disease among many other factors. Some beneficial therapeutic for knee osteoarthritis include weight loss, physical therapy, Tai Chi arts, needle taping, and most importantly exercise. $[12,13]$ The use of aquatic exercise has been shown to have good short term benefits. Also, massage therapy has been effective in managing osteoarthritis as recent trials have shown significantly favorable results. [14, 15] Nevertheless, a trial suggested that there is no significant benefit of physical therapy in hip osteoarthritis. [16]

Studies have shown that weight loss is recommended especially when the knees or hips are affected. [17] Although, a systemic review has found evidence of low quality about the effect of bariatric surgery on decreasing pain and morbidly in patients with knee involvement. [18] Some natural product supplements such as S-adenosylmethionine, methylsulfonylmethane, chondroitin, and glucosamine have been suggested in management but with variable effects on osteoarthritis management. Methylsulfonylmethane taken as three grams twice daily has been reported to significantly improve symptoms of pain and physical impairment. [19] However, more recent trials have shown that this effect is negligible over three months. [20] Other complementary therapy includes acupuncture, especially for knee and hip joint osteoarthritis. [21, 22]

\section{Medical Therapy}

For osteoarthritis, non-steroidal anti-inflammatory drugs (NSAIDs) are more effective than acetaminophen but considering which is safer, then acetaminophen is more appropriate when given in an adequate dose. However, the use of the non- 
steroidal anti-inflammatory drug for example diclofenac is most effective, succeeded by naproxen. [23] A somewhat effective medical therapy appearing in several small trials was topical capsaicin, although a noticed adverse effect is a transient burning sensation. [24] For moderate effect in treating osteoarthritis, tramadol shall be considered by the physician. [25] As for more severe and painful cases, duloxetine, which is a serotonin-norepinephrine reuptake inhibitor, is approved and shows a significant pain alleviation. [26] A common side effect in patients taking serotonin-norepinephrine reuptake inhibitors is moderate nausea. [27] Hence, only selected patients should use tramadol and duloxetine as they have similar side effects. Transdermal and oral opioids, with the exclusion of tramadol, have significant side effects and long term use is not recommended. Therefore, the family physician should predominantly start with acetaminophen at a full-dose with or without topical therapy. The general approach afterward is to use non-steroidal anti-inflammatory drugs (like diclofenac or naproxen), then, if needed, prescribe duloxetine or tramadol. Unfortunately, symptoms of chronic osteoarthritis tend to increment in severity, although they may temporarily improve in the short term with aggressive therapy. Corticosteroid injections have been shown to only relieve short term pain and decrease limitations, but they do not improve the quality of life. Furthermore, the steroidal injection has shown decrement in synovial fluid levels for ADULTS, a predictor of management success in osteoarthritis inflammation. [27]

\section{Surgical Therapy}

For patients with radiographic confirmation of osteoarthritis who suffer moderate or severe pain, surgery (joint replacement) may be indicated. It is good practice to have radiographic confirmation of knee osteoarthritis as this can lead to better management and a further improvement in function and pain control. Although, major complications can occur in these surgeries like infection, repeat surgery, or mobilization with the use of anesthesia and formation of deep vein thrombosis. [28] Often severe joint arthropathy would require hip or ankle joint replacement surgery, and factors such as daily life limitation and age are taken into consideration. An ineffective way for knee osteoarthritis is arthroscopic meniscectomy, which also showed insignificance in treating patients with degenerative meniscal tears. [29]

\section{Conclusion}

Osteoarthritis is a debilitating illness for which many patients follow-up their treatment alongside their general practitioner. The family physician should therefore attempt to discuss the different therapeutic options available including conservative, medical, and surgical interventions. Surgical procedures such as arthroscopic meniscectomy should be avoided in mild patients, and surgical approaches, in general, should be reserved for complicated resistant cases. Each patient has to receive their tailored management plan depending on a multifactorial basis.

\section{References}

1. Eldin MY, ElSayed WH, Abdulah GA, Abdalla HH. Effect of lateral wedged foot insole on Knee Proprioception in Knee Osteoarthritis. J. Adv. Pharm. Educ. Res. 2018;8(3): 18.

2. Saptarini N M, Hadisoebroto G, Wibowo M S, Gusdinar T. Effect of drug treatment on serum Cartilage Oligomeric Matrix Protein Levels in patients of Rheumatoid Arthritis and Knee Osteoarthritis in Bandung, Indonesia. J. Adv. Pharm. Educ. Res. 2020;10(2):41-5

3. Farhadian M, Morovati Z, Qafari Zadeh F, Shamsoddini A. The effect of Kinesio taping on the hand functions and symptoms in patients with hand osteoarthritis: a preliminary study. Int. j. pharm. phytopharm. res. 2019;9(4):75-80.

4. Aladwani S, Alosaimi ME, Althunayan SA, Khalaf A, Alrowaidan SM, Alhawas FA, Abdulaziz M, Aloyayri AA. A Survey to Assess Osteoporosis Knowledge of the General population of Riyadh, Saudi Arabia. Int. j. pharm. res. Allied sci. 2019;8(4):174-9.

5. Nelson AE, Jordan JM. Defining osteoarthritis: a moving target. Osteoarthritis Cartilage. 2012;20(1):1-3. doi:10.1016/j.joca.2011.10.008

6. Sacitharan PK. Aging and Osteoarthritis. Subcell Biochem. 2019;91:123-159. doi:10.1007/978-981-13-3681-2_6

7. Pereira D, Peleteiro B, Araújo J, Branco J, Santos RA, Ramos E. The effect of osteoarthritis definition on prevalence and incidence estimates a systematic review. Osteoarthritis Cartilage. 2011;19(11):1270-1285. doi:10.1016/j.joca.2011.08.009

8. Mobasheri A, Batt M. An update on the pathophysiology of osteoarthritis. Ann Phys Rehabil Med. 2016;59(5-6):333339. doi:10.1016/j.rehab.2016.07.004

9. Badley EM, Wilfong JM, Yip C, Millstone DB, Perruccio AV. The contribution of age and obesity to the number of painful joint sites in individuals reporting osteoarthritis: a population-based study. Rheumatology (Oxford). 2020;59(11):3350-3357. doi:10.1093/rheumatology/keaa138

10. Kohn MD, Sassoon AA, Fernando ND. Classifications in Brief: Kellgren-Lawrence Classification of Osteoarthritis. Clin Orthop Relat Res. 2016;474(8):1886-1893. doi:10.1007/s11999-016-4732-4

11. Karande SP, Kini S. Osteoarthritis: Clinical and Radiological Correlation. J Assoc Physicians India. 2018;66(7):37-39. 
12. Wang C, Schmid CH, Hibberd PL, Kalish R, Roubenoff R, Rones R, McAlindon T. Tai Chi is effective in treating knee osteoarthritis: a randomized controlled trial. Arthritis Care \& Research: Official Journal of the American College of Rheumatology. 2009; 61(11):1545-53. doi:10.1002/art.24832

13. Nahin RL, Boineau R, Khalsa PS, Stussman BJ, Weber WJ. Evidence-Based Evaluation of Complementary Health Approaches for Pain Management in the United States. Mayo Clin Proc. 2016;91(9):1292-1306. doi:10.1016/j.mayocp.2016.06.007

14. Perlman AI, Ali A, Njike VY, Hom D, Davidi A, Gould-Fogerite S, Milak C, Katz DL. Massage therapy for osteoarthritis of the knee: a randomized dose-finding trial. PLoS One. 2012;7(2):e30248. doi:10.1371/journal.pone.0030248

15. Perlman AI, Sabina A, Williams AL, Njike VY, Katz DL. Massage therapy for osteoarthritis of the knee: a randomized controlled trial. Arch Intern Med. 2006;166(22):2533-2538. doi:10.1001/archinte.166.22.2533

16. Bennell KL, Egerton T, Martin J, Abbott JH, Metcalf B, McManus F, Sims K, Pua YH, Wrigley TV, Forbes A, Smith C. Effect of physical therapy on pain and function in patients with hip osteoarthritis: a randomized clinical trial. Jama. 2014 May 21;311(19):1987-97. doi:10.1001/jama.2014.4591

17. Gay C, Chabaud A, Guilley E, Coudeyre E. Educating patients about the benefits of physical activity and exercise for their hip and knee osteoarthritis. Systematic literature review. Ann Phys Rehabil Med. 2016;59(3):174-183. doi:10.1016/j.rehab.2016.02.005

18. Groen VA, van de Graaf VA, Scholtes VA, Sprague S, van Wagensveld BA, Poolman RW. Effects of bariatric surgery for knee complaints in (morbidly) obese adult patients: a systematic review. Obes Rev. 2015;16(2):161-170. doi:10.1111/obr.12236

19. Kim LS, Axelrod LJ, Howard P, Buratovich N, Waters RF. Efficacy of methylsulfonylmethane (MSM) in osteoarthritis pain of the knee: a pilot clinical trial. Osteoarthritis Cartilage. 2006;14(3):286-294. doi:10.1016/j.joca.2005.10.003

20. Debbi EM, Agar G, Fichman G, Ziv YB, Kardosh R, Halperin N, Elbaz A, Beer Y, Debi R. Efficacy of methylsulfonylmethane supplementation on osteoarthritis of the knee: a randomized controlled study. BMC complementary and alternative medicine. 2011 Dec 1;11(1):50. Published 2011 Jun 27. doi:10.1186/1472-6882-11-50

21. Wu N, Huang J, Yang X, Guo J, Liu F, Gu Y, Liu Y, Zhang Z, Yin S, Feng X. The long-term effect of acupuncture for patients with knee osteoarthritis: Study protocol for a randomized controlled trial. Medicine. 2020 Oct 16;99(42). doi:10.1097/MD.0000000000022599

22. Chan SC, Engkasan P. Does acupuncture improve pain and function in people with hip osteoarthritis? A Cochrane Review summary with commentary. International Journal of Rheumatic Diseases. 2020 Oct 29. doi:10.1111/1756$185 X .13948$

23. da Costa BR, Reichenbach S, Keller N, Nartey L, Wandel S, Jüni P, Trelle S. Effectiveness of non-steroidal antiinflammatory drugs for the treatment of pain in knee and hip osteoarthritis: a network meta-analysis. The Lancet. 2017 Jul 8;390(10090):e21-33. doi:10.1016/S0140-6736(17)31744-0

24. De Silva V, El-Metwally A, Ernst E, Lewith G, Macfarlane GJ. Arthritis Research UK Working Group on Complementary and Alternative Medicines. Evidence for the efficacy of complementary and alternative medicines in the management of osteoarthritis: a systematic review. Rheumatology (Oxford). 2011;50(5):911-20. doi:10.1093/rheumatology/keq379.

25. Cepeda MS, Camargo F, Zea C, Valencia L. Tramadol for osteoarthritis: a systematic review and metaanalysis. The Journal of rheumatology. 2007 Mar 1;34(3):543-55.

26. Wang ZY, Shi SY, Li SJ, Chen F, Chen H, Lin HZ, Lin JM. Efficacy and safety of duloxetine on osteoarthritis knee pain: a meta-analysis of randomized controlled trials. Pain Medicine. 2015 Jul 1;16(7):1373-85. doi:10.1111/pme.12800.

27. Brunton S, Wang F, Edwards SB, Crucitti AS, Ossanna MJ, Walker DJ, Robinson MJ. Profile of adverse events with duloxetine treatment. Drug safety. 2010 May 1;33(5):393-407. doi:10.2165/11319200-000000000-00000.

28. Skou ST, Roos EM, Laursen MB, Rathleff MS, Arendt-Nielsen L, Simonsen O, Rasmussen S. A randomized, controlled trial of total knee replacement. New England Journal of Medicine. 2015 Oct 22;373(17):1597-606. doi:10.1056/NEJMoa1505467.

29. Nakagawa S, Arai Y, Inoue H, Fujii Y, Kaihara K, Mikami Y. Relationship of alignment in the lower extremity with early degeneration of articular cartilage after resection of the medial meniscus: Quantitative analysis using T2 mapping. Medicine (Baltimore). 2020;99(44):e22984. doi:10.1097/MD.0000000000022984. 